\title{
Supplementary Material: MD Simulations of Spontaneous Membrane Protein/Detergent Micelle Formation
}

Simulation system details: To generate the starting configurations for the spontaneous self-association simulations, 80 or 60 DPC detergents were placed at random positions in boxes surrounding a central OmpA or GpA molecule, respectively. Starting structures for the pre-formed micelles were constructed with the same number of detergent molecules using a previously described protocol ${ }^{1}$. Briefly, the micelles consisted of semicircular planes of DPC detergents in the all-trans configuration, packed in a toroidal fashion around the hydrophobic surface of each protein. Starting protein coordinates corresponded to crystal structure PDB file 1 bxw for OmpA ${ }^{2}$ (resolution $2.5 \AA$ ), and to the solid state NMR structure for $\mathrm{GpA}^{3}$. In each case, the DPC concentration was $\sim 0.2 \mathrm{M}$. During each simulation, protein structural stability was maintained, as monitored by the root mean square deviation from the initial structure, in particular for the secondary-structural elements (Table 1). All simulations were performed using the GROMACS simulation package ${ }^{4}$. An extended united atom version of the GROMOS96 force field ${ }^{5}$ was used. The DPC charges were taken from ${ }^{6}$. Every system was energy minimized before $\mathrm{MD}$, using $<1000$ steps of the steepest descent method in order to relax any steric conflict(s) generated during the set-up. Each system was solvated with SPC waters and sufficient counter ions to make the system electically neutral, with a final concentration of $\sim 0.1 \mathrm{M} \mathrm{NaCl}$. Brief $\sim 1$ ns equilibration runs were carried out for the pre-formed micelle systems, to allow DPC molecules to pack around each protein. During these runs, protein was harmonically restrained with a force constant of $1000 \mathrm{~kJ} \mathrm{~mol}^{-1} \mathrm{~nm}^{-2}$. In all simulations, long range electrostatic interactions were calculated using the particle mesh Ewald method with a $1 \mathrm{~nm}$ cut-off for the real space calculation ${ }^{7}$. A cutoff of $1 \mathrm{~nm}$ was used for the van der Waals interactions. All simulations were performed at constant temperature, pressure and number of particles. The temperature of the protein, detergent, and solvent were each coupled separately, using the Berendsen thermostat ${ }^{8}$ at 300 $\mathrm{K}$ with a coupling constant of $\tau_{\mathrm{T}}=0.1 \mathrm{ps}$ for the OmpA simulations, and using the Nosé-Hoover thermostat ${ }^{9,10}$ at $313 \mathrm{~K}$ with a coupling constant of $\tau_{\mathrm{T}}=0.5 \mathrm{ps}$ for the GpA simulations. The pressure was coupled at 1 bar with compressibility $=4.5 \times 10^{-5}$ bar $^{-1}$, using the Berendsen barostat with coupling constant $\tau_{\mathrm{P}}=1$ ps for the OmpA simulations, and using the Parrinello-Rahman barostat ${ }^{11,12}$ with coupling constant $\tau_{\mathrm{P}}=5$ ps for the GpA simulations. The LINCS algorithm ${ }^{13}$ was used throughout to restrain bond lengths. The timestep for integration was 2 fs for the GpA simulations and for the OmpA pre-formed micelle simulation. An integration timestep of 5 fs was used for the OmpA self-association simulation to increase simulation efficiency. This larger timestep is possible with a special treatment of hydrogen atoms ${ }^{6,14}$. Simulations were performed on dual Pentium 3 Linux workstations, or on a 68-node Linux cluster composed of dual Xeon 4 processors. Analyses were performed using GROMACS tools and/or locally-written code.

Table S1: Details of simulation setups.

\begin{tabular}{|l|l|l|l|l|l|l|}
\hline System & $\begin{array}{l}\text { Simulation } \\
\text { length (ns) }\end{array}$ & $\begin{array}{l}\text { No. of water } \\
\text { molecules }\end{array}$ & No. of atoms & $\begin{array}{l}\text { Approximate } \\
\text { box size (nm) }\end{array}$ & $\begin{array}{l}\text { RMSD } \\
\text { secondary structure } \\
\text { (nm) (last 10 ns) }\end{array}$ & $\begin{array}{l}\text { RMSD for non- } \\
\text { secondary } \\
\text { (nm) (last 10 ns) }\end{array}$ \\
\hline $\begin{array}{l}\text { Self-assembly.: } \\
\text { OmpA+80DPC }\end{array}$ & 50 & 29,395 & 91,871 & $9 \times 9 \times 12$ & $0.15 \pm 0.01$ \\
\hline $\begin{array}{l}\text { Pre-formed: } \\
\text { OmpA+80DPC }\end{array}$ & 25 & 19,094 & 60,914 & $9 \times 9 \times 8$ & $0.11 \pm 0.01$ \\
\hline $\begin{array}{l}\text { Self-assembly: } \\
\text { GpA+60DPC }\end{array}$ & 50 & 14,783 & 46,193 & $9 \times 9 \times 6.5$ & $0.12 \pm 0.02$ \\
\hline $\begin{array}{l}\text { Pre-formed: } \\
\text { GpA+60DPC }\end{array}$ & 50 & 14,960 & 46,724 & $9 \times 9 \times 6.5$ & $0.62 \pm 0.03$ \\
\hline
\end{tabular}

Supplementary Analysis of Simulations: A more detailed comparative analysis reveals that the protein/detergent micelles at the end of the self-assembly simulations are very similar to those at the end of the simulations initiated from pre-formed micelles. Thus, in Fig. S1 it can be seen that the radial density profiles for the self-assembled vs. pre-formed micelles are very similar, remarkably so for the GpA simulations. This provides a further degree of confidence that the self-assembly simulations have 'converged' in the sense of yielding a stable micelle structure comparable to that constructed 'by hand'.

Various analyses provide further characterization of the kinetics of the process of micelle formation. In Fig. S2 the detergent headgroupheadgroup interactions are examined as a function of time. For both simulations, the average number of detergent phosphate atoms surrounding one another reaches the equilibrium value of $\sim 3$ after $\sim 10 \mathrm{~ns}$, similar to the values in the pre-formed micelle simulatons. Analysis of the diffusion coefficients of the detergent molecules (Fig. S3) reveals an exponential decline to a common equilibrium value. Interestingly, the ratio of the time constants for decay of the detergent diffusion coefficients was $\sim 2: 1$ for OmpA:GpA, the same as the ratio for the decay in radius of gyration of the two micelles (see Fig. 2 of main text). Examination of the solvent-accessible surface areas of the detergent molecules as a function of time (Fig. S4) reveals that interactions of the detergent headgroups relax more quickly than those of the tails. The proportion of protein solvent-accessible surface area (as a function of the total protein surface area) for both simulations declines significantly over the first 10 ns followed by subsequent slower relaxation (Fig. S5). The trend in decline is similar for each protein structural group, further supporting a model of simple, diffusional adsorption (see main text for details) during micelle formation." 

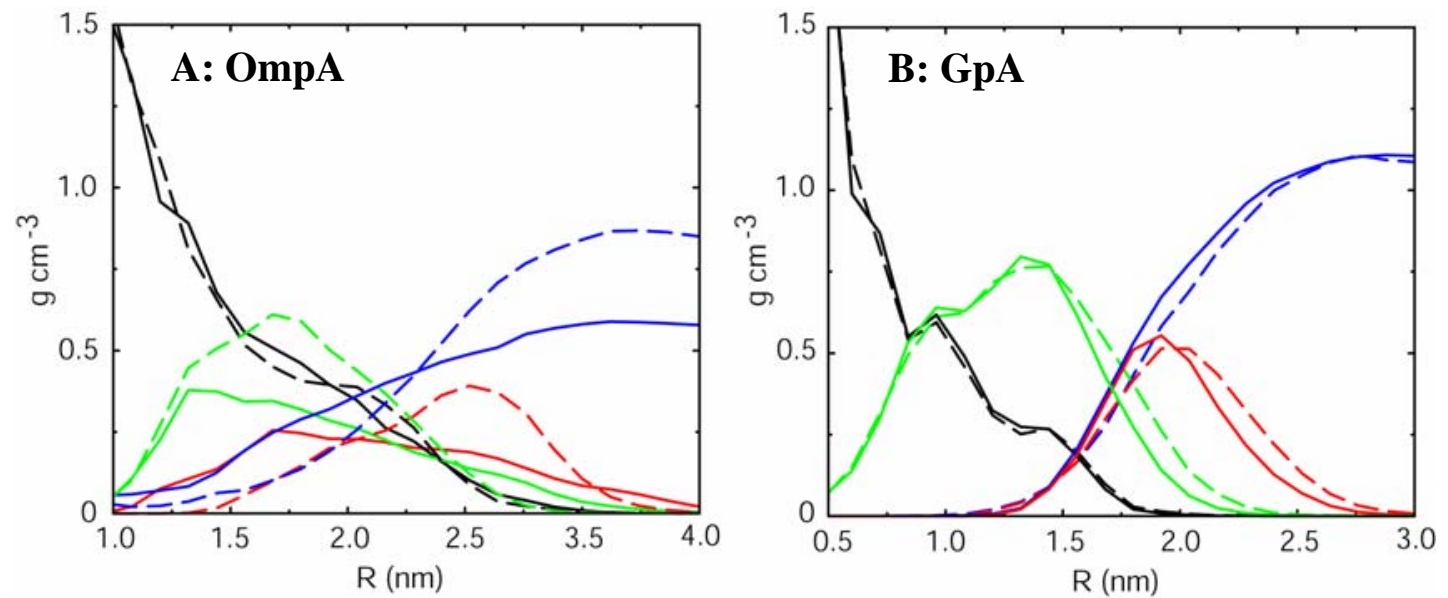

Figure S1: Radial density relative to centre of mass of protein for protein (black), water (blue), DPC tails (green), and DPC headgroups (red) for self-assembled (solid lines) and pre-formed (dashed lines) micelles for OmpA (A) and GpA (B) simulations.
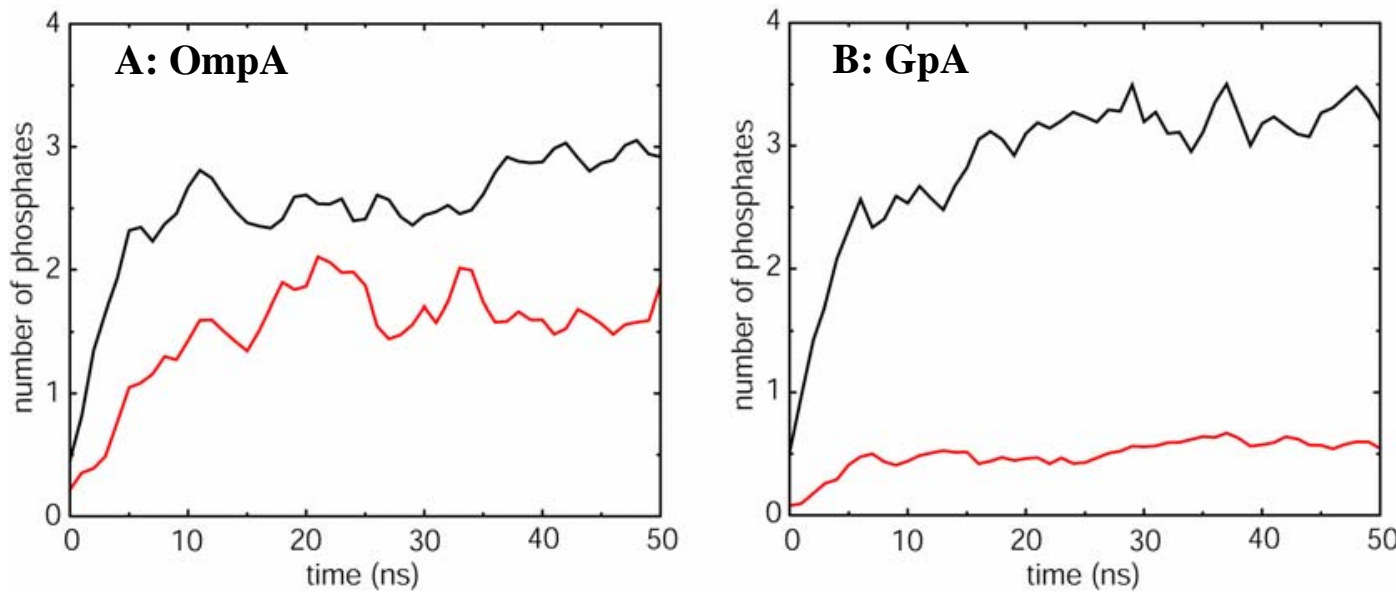

Figure S2: Average number of detergent phosphate atoms surrounding one another in the first "shell" over time, for the (A) OmpA and (B) GpA simulations. These values were determined by integrating the P-P radial distribution function (calculated with a $0.05 \mathrm{~nm}$ bin-width) to the first minimum $(1 \mathrm{~nm})$ in $1 \mathrm{~ns}$ intervals. The P-P shell numbers were calculated for interactions within the main protein/detergent micelle (black lines), and for interactions between the main micelle and the globule (red lines). Average P-P intra-micelle shell numbers for last 10 ns of the equivalent pre-formed micelle simulations were both $\sim 3$.
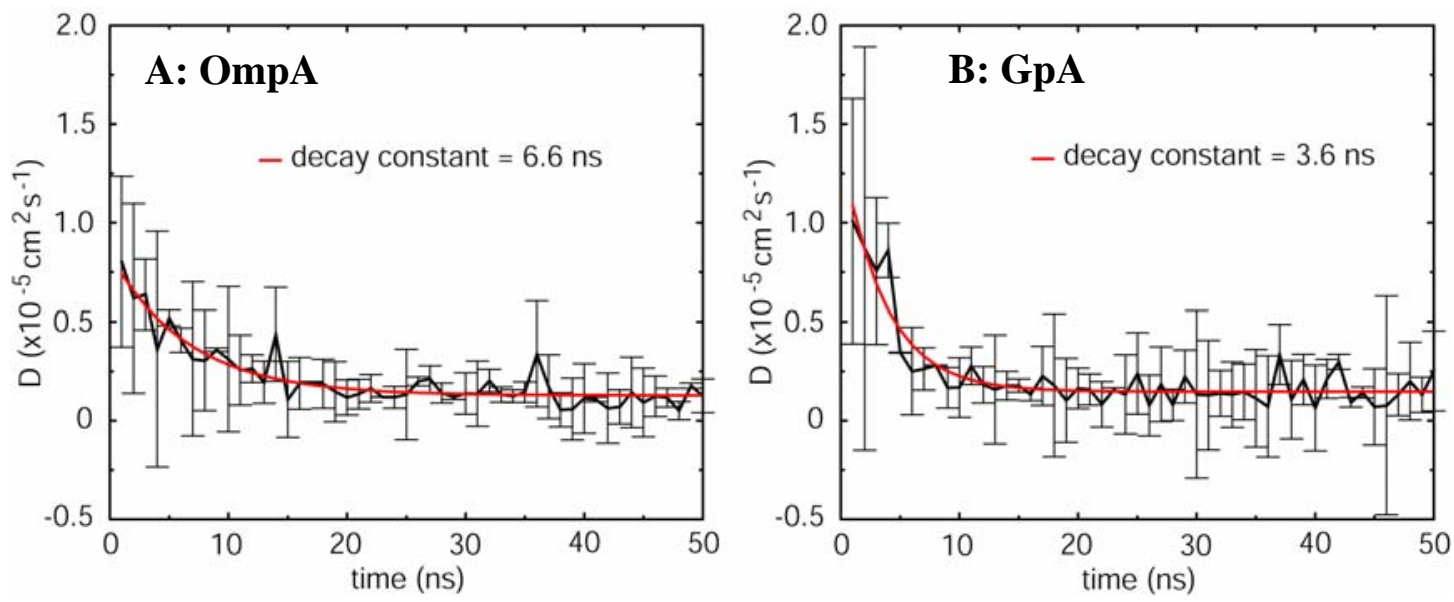

Figure S3: Average diffusion (black lines) of all detergent molecules over time for the (A) OmpA and (B) GpA simulations. Exponential fits (red lines) resulted in decay constants of $6.6 \mathrm{~ns}$ for OmpA and $3.6 \mathrm{~ns}$ for GpA, yielding a ratio of $\sim 2$. Average MSD values for the last $10 \mathrm{~ns}$ of both of the equivalent pre-formed micelle simulations were both $\sim 0.1 \times 10^{-5} \mathrm{~cm}^{2} \mathrm{~s}^{-1}$. Diffusion coefficients were calculated in $1 \mathrm{~ns}$ intervals using the Einstein relation by least squares fitting a straight line through the mean square displacement (MSD) curves. Error bars indicate the difference of the diffusion coefficients obtained from fits over the two halves of the 1 ns fit intervals. 

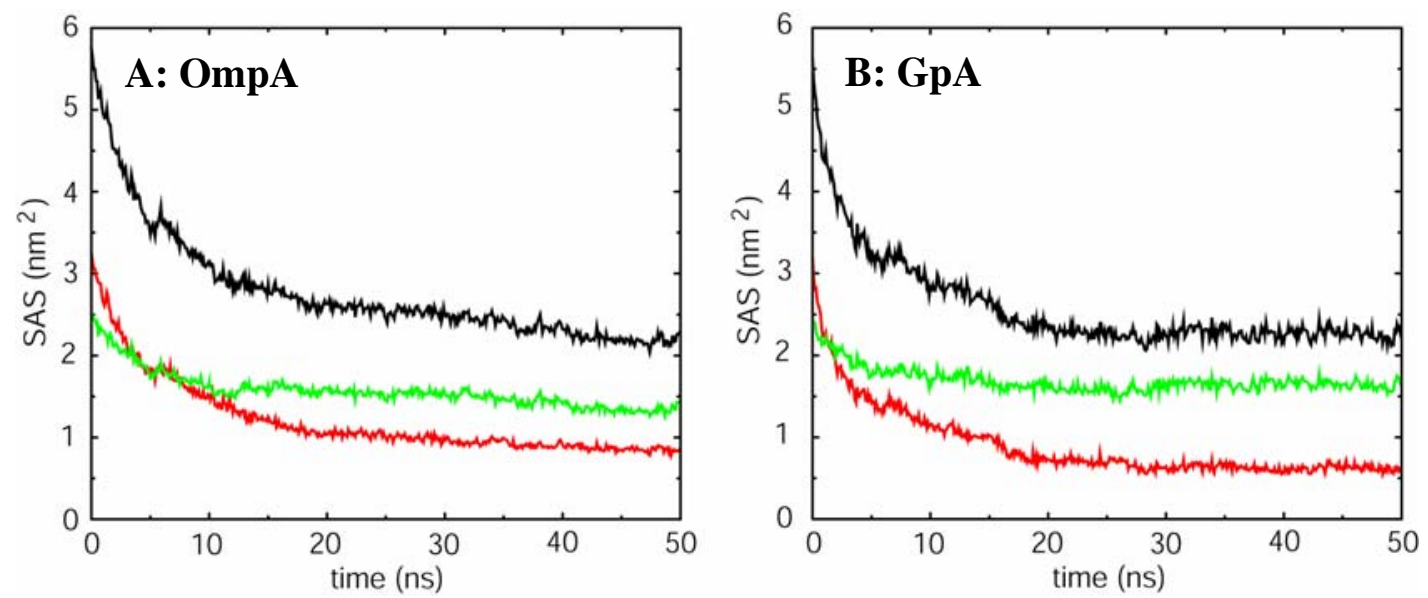

Figure S4: Detergent solvent-accessible surface area (SAS) per detergent molecule over time, for all detergents of the main protein/detergent aggregate, excluding the globules, for the (A) OmpA and (B) GpA simulations. The SAS per detergent is shown for the entire detergent molecules (black lines), for the acyl tail regions (red lines), and for the polar headgroup regions (green lines). Average SAS per detergent for the last $10 \mathrm{~ns}$ of the equivalent pre-formed OmpA micelle simulation were $\sim 1.9$ (total), $\sim 0.4$ (tails), and $\sim 1.4$ (heads) $\mathrm{nm}^{2}$, and for GpA were $\sim 2.1$ (total), $\sim 0.6$ (tails), and $\sim 1.5$ (heads) $\mathrm{nm}^{2}$.
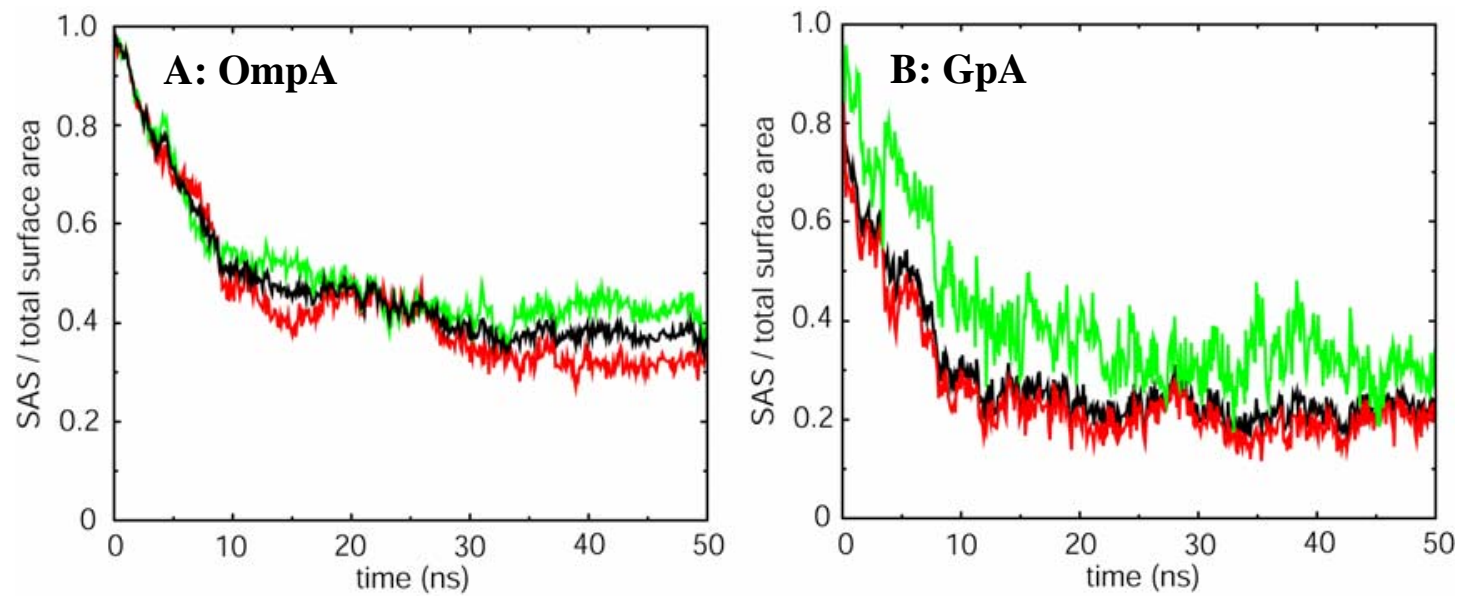

Figure S5: Protein solvent-accessible surface area (SAS) divided by the total protein surface area, over time, for the (A) OmpA and (B) GpA simulations. SAS is indicated for the whole protein (black lines), for the regions of secondary structure (SS) (red lines), and for the regions of non-secondary structure (NSS) (green lines). Average values for the last 10 ns of the equivalent pre-formed OmpA micelle simulation were $\sim 0.4$ (total), $\sim 0.2$ (SS), and $\sim 0.6$ (NSS), and for GpA were $\sim 0.2$ (total), 0.2 (SS), and $~ 0.3$ (NSS).

\section{References:}

(1) Bond, P. J.; Sansom, M. S. P., Membrane protein dynamics versus environment: simulations of OmpA in a micelle and in a bilayer. J. Mol. Biol. 2003, 329, 1035-1053.

(2) Pautsch, A.; Schulz, G. E., Structure of the outer membrane protein A transmembrane domain. Nature Struct. Biol. 1998, 5, 1013-1017.

(3) Smith, S. O.; Song, D.; Shekar, S.; Groesbeek, M.; Ziliox, M.; Aimoto, S., Structure of the transmembrane dimer of glycophorin A in membrane bilayers. Biochem. 2001, 40, (22), 6553-6558

(4) Lindahl, E.; Hess, B.; Van der Spoel, D., GROMACS 3.0: a package for molecular simulation and trajectory analysis. J. Mol. Model. 2001, 7, 306-317.

(5) Van Gunsteren, W. F.; Kruger, P.; Billeter, S. R.; Mark, A. E.; Eising, A. A.; Scott, W. R. P.; Huneberger, P. H.; Tironi, I. G., Biomolecular Simulation: The GROMOS96 Manual and User Guide. ed.; Biomos \& Hochschulverlag AG an der ETH Zurich: Groningen \& Zurich, 1996.

(6) Tieleman, D. P.; van der Spoel, D.; Berendsen, H. J. C., Molecular dynamics simulations of dodecylphosphocholine micelles at three different aggregate sizes: Micellar structure and chain relaxation. J. Phys. Chem. B 2000, 104, 6380-6388.

(7) Darden, T.; York, D.; Pedersen, L., Particle mesh Ewald - an N.log(N) method for Ewald sums in large systems. J. Chem. Phys. 1993, 98, 10089-10092. 
(8) Berendsen, H. J. C.; Postma, J. P. M.; van Gunsteren, W. F.; DiNola, A.; Haak, J. R., Molecular dynamics with coupling to an external bath. J. Chem. Phys. 1984, 81, 3684-3690.

(9) Nose, S., A molecular dynamics method for simulations in the canonical ensemble. Mol. Phys. 1984, 52, 255-268.

(10) Hoover, W. G., Canonical dynamics: equilibrium phase-space distributions. Phys. Rev. 1985, A31, $1695-1697$.

(11) Parrinello, M.; Rahman, A., Polymorphic Transitions in Single-Crystals - a New Molecular- Dynamics Method. J. Appl. Phys. 1981, 52, 7182-7190.

(12) Nose, S.; Klein, M. L., Constant Pressure Molecular-Dynamics for Molecular-Systems. Mol. Phys. 1983, 50, $1055-1076$.

(13) Hess, B.; Bekker, H.; Berendsen, H. J. C.; Fraaije, J., LINCS: A linear constraint solver for molecular simulations. J. Comp. Chem. 1997, 18, 1463-1472.

(14) Feenstra, K. A.; Hess, B.; Berendsen, H. J. C., Improving efficiency of large time-scale molecular dynamics simulations of hydrogen-rich systems. J. Comp. Chem. 1999, 20, 786-798. 